\title{
Coherent cyclotron motion beyond Kohn's theorem
}

\author{
T. Maag', A. Bayer', S. Baierl', M. Hohenleutner', T. Korn' ', C. Schüller', D. Schuh', D. Bougeard1, \\ C. Lange ${ }^{1 \star}$, R. Huber ${ }^{1}$, M. Mootz ${ }^{2}$, J. E. Sipe ${ }^{2,3}$, S. W. Koch ${ }^{2}$ and M. Kira ${ }^{2}$
}

In solids, the high density of charged particles makes manybody interactions a pervasive principle governing optics and electronics $^{1-12}$. However, Walter Kohn found in 1961 that the cyclotron resonance of Landau-quantized electrons is independent of the seemingly inescapable Coulomb interaction between electrons ${ }^{2}$. Although this surprising theorem has been exploited in sophisticated quantum phenomena ${ }^{13-15}$, such as ultrastrong light-matter coupling ${ }^{16}$, superradiance ${ }^{17}$ and coherent control ${ }^{18}$, the complete absence of nonlinearities excludes many intriguing possibilities, such as quantum-logic protocols ${ }^{19}$. Here, we use intense terahertz pulses to drive the cyclotron response of a two-dimensional electron gas beyond the protective limits of Kohn's theorem. Anharmonic Landau ladder climbing and distinct terahertz four- and six-wave mixing signatures occur, which our theory links to dynamic Coulomb effects between electrons and the positively charged ion background. This new context for Kohn's theorem unveils previously inaccessible internal degrees of freedom of Landau electrons, opening up new realms of ultrafast quantum control for electrons.

Controlling superpositions of electronic quantum states has been a paradigm of fundamental physics and quantum-information technology ${ }^{19}$. Sophisticated protocols have been implemented in atomic gases ${ }^{20}$, whereas solids represent a more challenging environment owing to complicated many-body interactions. Quantum manipulation has been fairly successful in atom-like single-particle systems, such as quantum dots ${ }^{21}$, whereas controlling interacting many-body systems is still in its infancy. Following Lev Landau's suggestion to combine collective properties of an interacting particle system into quasiparticles ${ }^{1}$, researchers effectively analyse crystal electrons and holes ${ }^{4,5}$, excitons ${ }^{6,9}$, dropletons $s^{11}$, polarons ${ }^{7}$, magnons $s^{22}$ or Cooper pairs ${ }^{12}$. In particular, ultrashort pulses in the terahertz $\left(1 \mathrm{THz}=10^{12} \mathrm{~Hz}\right)$ spectral range have become a powerful tool to probe $e^{4,6,7,12,17}$ and control ${ }^{8,18,22,23}$ quasiparticle excitations, such as intra-excitonic transitions ${ }^{6}$ or superconductor Higgs bosons ${ }^{12}$.

For most quasiparticle excitations, Coulomb scattering leads to coherence times in the range of a few to a few hundred femtoseconds, which is too short for most quantum-logic operations. However, the cyclotron resonance (CR) in a two-dimensional electron gas (2DEG) represents a unique exception ${ }^{2,13-18}$. In a magnetically biased 2DEG, Landau electrons emerge as elementary quasiparticles. According to Kohn's theorem the quantization energy of the harmonic spectrum of Landau levels (LLs) defined by the cyclotron frequency $v_{c}$ is unaffected by Coulomb scattering ${ }^{2}$. Although the a
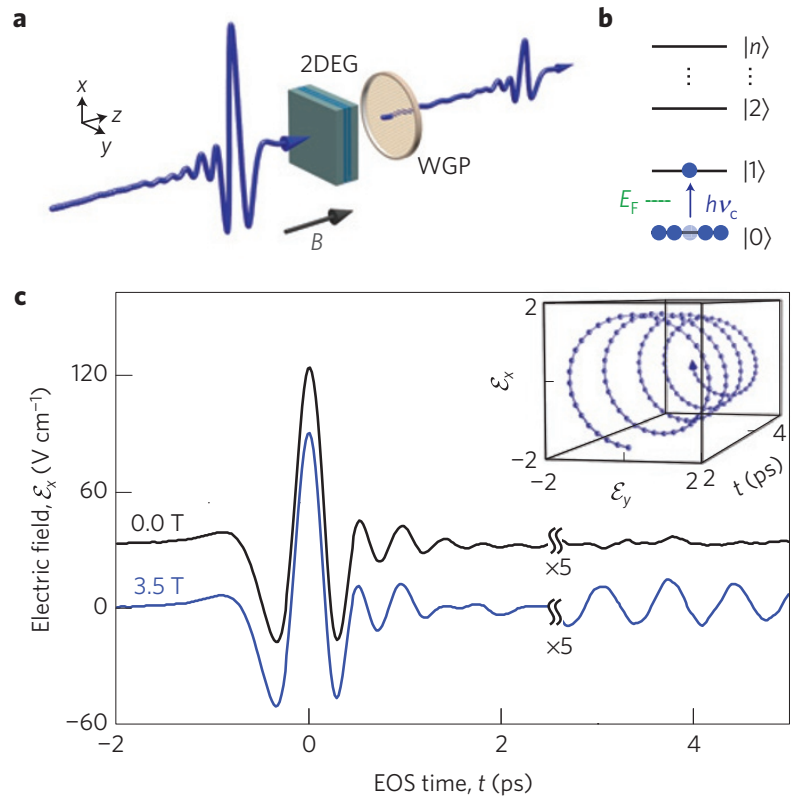

Figure 1 | Linear terahertz magnetospectroscopy of a 2DEG Landau system. a, Schematic of time-domain terahertz set-up in Faraday geometry. Linearly polarized terahertz transients drive inter-Landau level transitions in two 30-nm wide, n-doped GaAs QWs. The transmitted waveforms are detected via electro-optic sampling (EOS) in a ZnTe crystal with polarization selectivity achieved through a wire-grid polarizer (WGP). b, Landau level scheme. At $B=3.5 \mathrm{~T}$, all electrons condense to the first Landau state $|n=0\rangle$ with a filling factor of $f=0.95$. c, Transmitted terahertz pulses at $B=3.5 \mathrm{~T}$ (blue curve) and $B=0 \mathrm{~T}$ (black curve, offset for clarity). For $B=3.5 \mathrm{~T}$, trailing oscillations result from the decay of the polarization, which our low-field terahertz transient excites solely between $\operatorname{LLs}|n=0\rangle$ and $|n=1\rangle$. Inset: Polarization-resolved plot of the re-emission showing its circular nature.

absence of Coulomb complications warrants a long-lived CR coherence, the accompanying perfectly harmonic behaviour has an apparent downside: multiphoton excitations drive perfect Landau electrons only to climb up the LLs, preventing Rabi flopping, which is the desired elementary process in many quantumlogic operations ${ }^{19}$.

Here, we investigate the possibility of inducing a distinctly anharmonic CR response with the goal of coherently controlling transitions among a few selected LLs. Intense terahertz pulses coherently

\footnotetext{
${ }^{1}$ Department of Physics, University of Regensburg, 93040 Regensburg, Germany. ${ }^{2}$ Department of Physics, University of Marburg, 35032 Marburg, Germany. ${ }^{3}$ Department of Physics and Institute for Optical Sciences, University of Toronto, 60 St George St., Toronto, Ontario M5S 1A7, Canada. *e-mail: christoph.lange@physik.uni-regensburg.de
} 


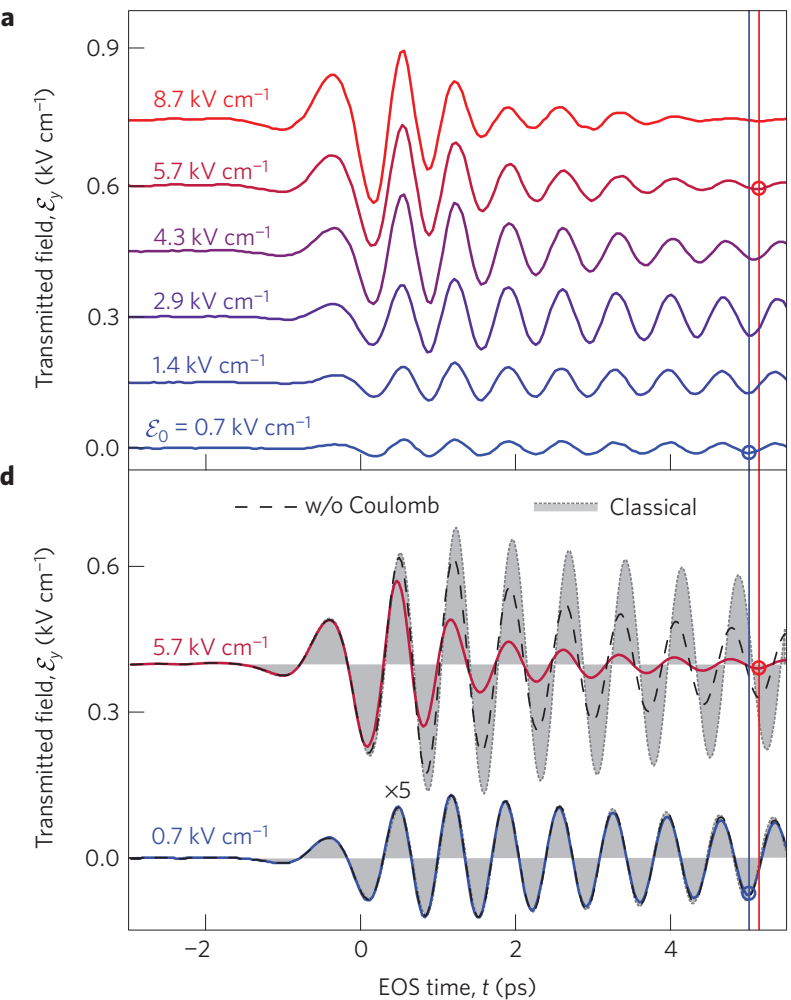

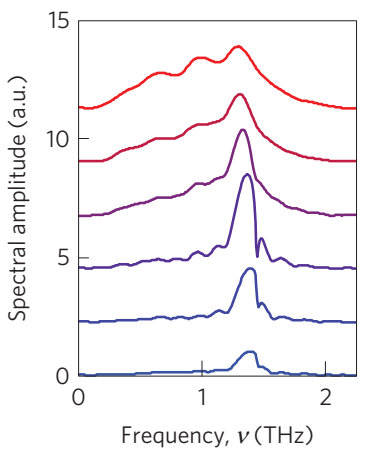

c

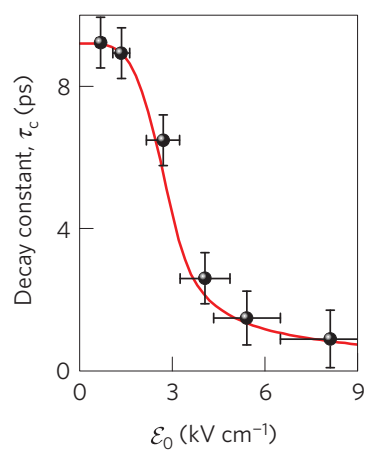

e

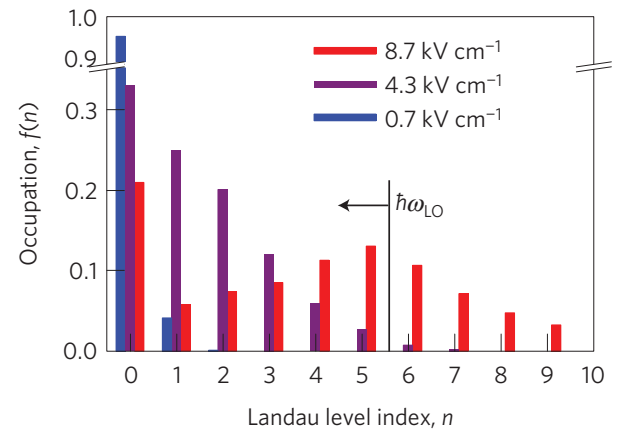

Figure 2 | Dynamics of Landau system under non-perturbative single-pulse excitation. a, Waveform of the transmitted field $\mathcal{E}_{y}$ for various amplitudes $\mathcal{E}_{0}$ of the incident terahertz transient (polarized in $x$-direction), vertically offset for clarity. Vertical lines indicate the temporal position of a local minimum of $\mathcal{E}_{y}$ for low-field excitation ( $\mathcal{E}_{0}=0.7 \mathrm{kV} \mathrm{cm}^{-1}$, vertical blue line) and high-field excitation $\left(\mathcal{E}_{0}=5.7 \mathrm{kV} \mathrm{cm}^{-1}\right.$, vertical red line), indicating a field-induced phase retardation. $\mathbf{b}$, Fourier transform of $\mathcal{E}_{y}$ from a evidencing the broadening and redshift of the CR from 1.45 to 1.3 THz. $\mathbf{c}$, Coherence time $\tau_{\mathrm{c}}$ extracted from fitting the waveforms $\mathcal{E}_{y}(t)$ in a with an exponentially decaying sinusoidal function (black dots), and calculation (red line). Vertical and horizontal error bars mark the standard deviation intervals $( \pm \sigma)$ for the fit and for the terahertz amplitude, respectively, the latter arising from uncertainties in the terahertz spot size and power. $\mathbf{d}$, Terahertz dynamics calculated with a full quantum theory for $\mathcal{E}_{0}=0.7 \mathrm{kV} \mathrm{cm}^{-1}(\mathrm{blue} \mathrm{curve})$ and $\mathcal{E}_{0}=5.7 \mathrm{kV} \mathrm{cm}^{-1}($ red

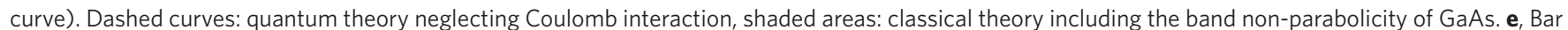
chart of calculated Landau level population as a function of $\mathrm{LL}$ index $n$ at a fixed delay time $t=0.6 \mathrm{ps}$, for $\mathcal{E}_{0}=0.7 \mathrm{kV} \mathrm{cm}^{-1}\left(\mathrm{blue}\right.$ bars), $\mathcal{E}_{0}=4.3 \mathrm{kV} \mathrm{cm}^{-1}$ (violet bars) and $\mathcal{E}_{0}=8.7 \mathrm{kV} \mathrm{cm}^{-1}$ (red bars). Phonon scattering sets in for energies above the LO phonon energy $\hbar \omega_{\mathrm{LO}}$ (vertical black line and arrow).

drive the state of a 2 DEG up the Landau ladder by as much as six rungs within a single cycle of the carrier wave, and induce anharmonicities which render LL transitions distinguishable. Furthermore, strong coherent nonlinearities, such as four- and six-wave mixing signals are observed. Although Kohn's theorem has been violated via phonons ${ }^{24,25}$, non-parabolic electron dispersion ${ }^{26}$, or in a Bose-Einstein condensate close to a Feshbach resonance ${ }^{27}$, our experiment-theory comparison unambiguously reveals a nonlinear response that stems from the Coulomb interaction itself, between the electrons and the ionic background.

Our sample hosts two 30-nm wide n-doped GaAs quantum wells (QWs; see Methods), each containing a 2DEG. To calibrate the system, phase-locked low-amplitude $\left(\mathcal{E}_{0}=90 \mathrm{~V} \mathrm{~cm}^{-1}\right)$ terahertz pulses are transmitted through the structure. Polarization components parallel $\left(\mathcal{E}_{x}\right)$ and perpendicular $\left(\mathcal{E}_{y}\right)$ to the incident pulses are separately detected by electro-optic sampling (EOS), as a function of delay time $t$ (Fig. 1a). Without magnetic bias, $\mathcal{E}_{x}(t)$ (Fig. 1c, black curve) closely follows the incident waveform (Supplementary Discussion 1). When a magnetic bias $B=3.5 \mathrm{~T}$ is applied perpendicularly to the 2DEG plane, long-lived trailing oscillations emerge owing to the CR with $v_{\mathrm{c}}=1.45 \mathrm{THz}$ (Fig. 1c, blue curve $)^{18}$. Here the weak terahertz field induces a polarization between LLs $|n=0\rangle$ (filling factor $f=0.95$ ) and $|n=1\rangle$ (Fig. 1b), reemitting a circularly polarized terahertz wave (Fig. 1c, inset) whose coherence time $\tau_{c}=9 \mathrm{ps}$ is probably limited by superradiant decay ${ }^{17}$.

We then drive the calibrated system with intense terahertz pulses generated by tilted-pulse-front optical rectification ${ }^{23}$. The amplitude of the re-emitted field $\mathcal{E}_{y}(t)$ (Fig. 2a) increases with $\mathcal{E}_{0}$, but the temporal waveform remains similar up to $\mathcal{E}_{0}=1.4 \mathrm{kV} \mathrm{cm}^{-1}$. For $\mathcal{E}_{0} \geq 2.9 \mathrm{kV} \mathrm{cm}^{-1}$, the trailing oscillations slow down and decay more rapidly until the inter-LL polarization essentially follows the driving field, for $\mathcal{E}_{0}=8.7 \mathrm{kV} \mathrm{cm}^{-1}$, as the coherence decays almost instantly. The spectrum of the re-emitted field (Fig. 2b), which is initially centred at $v_{c}=1.45 \mathrm{THz}$, redshifts and broadens with increasing $\mathcal{E}_{0}$ until its shape converges to the spectrum of the driving pulse. This pronounced field dependence is irreconcilable with the linear response of a harmonic oscillator, indicating that the cyclotron transition is driven into a strongly nonlinear regime by the intense terahertz transients. Figure $2 \mathrm{c}$ shows the dephasing time $\tau_{\mathrm{c}}$ as a function of the terahertz amplitude $\mathcal{E}_{0}$. Starting at a low-field value of $\tau_{c}=9 \mathrm{ps}$, the decay time drops abruptly for $\mathcal{E}_{0}>3 \mathrm{kV} \mathrm{cm}^{-1}$, and ultimately approaches $\tau_{\mathrm{c}}=0.5 \mathrm{ps}$.

Kohn's theorem implies that the purely repulsive electronelectron Coulomb interaction cannot produce observable nonlinearities in a 2DEG. However, in a real modulation-doped system, the 2DEG always resides on a positive background charge that holds the electrons together as a homogeneous gas by preventing the formation of a Wigner crystal ${ }^{28}$ or the escape of electrons to the edges of the sample. As the attractive electron-ion interaction is not limited by Kohn's theorem, it can indeed induce CR nonlinearities.

The experiments are analysed with cluster-expansion-based many-body computations ${ }^{3}$ including the non-parabolic dispersion of Landau electrons, their coupling to terahertz fields as well as phonons, and the Coulombic electron-electron and electron-ion 
a

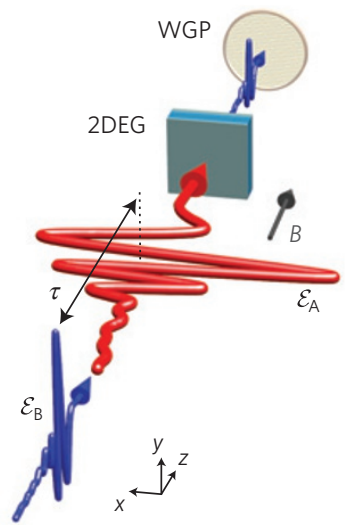

\section{b}
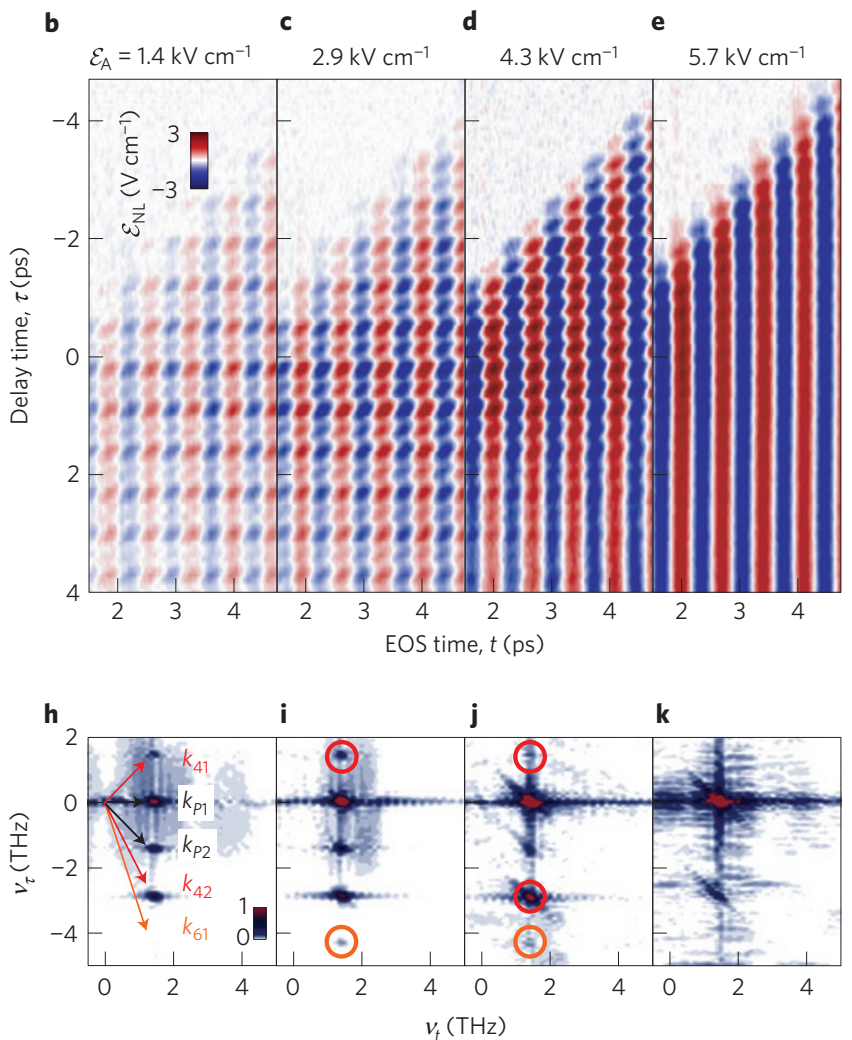
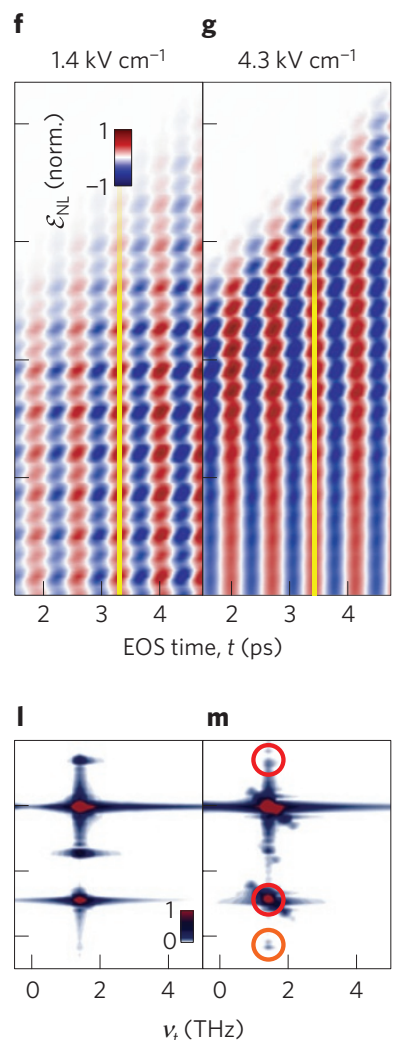

Figure 3 | Two-dimensional, phase-resolved, collinear terahertz spectroscopy. a, Schematic: Strong terahertz pulses $A$ of amplitude $\mathcal{E}_{\mathrm{A}}$ (polarized in $x$-direction) prepare a coherent inter-LL polarization in the $2 D E G$. The nonlinear terahertz response $\mathcal{E}_{\mathrm{NL}}(t, \tau)$ is time-resolved using weak pulses $B$ of amplitude $\mathcal{E}_{\mathrm{B}}=90 \mathrm{~V} \mathrm{~cm}^{-1}$ (polarized in $y$-direction), incident after a relative delay time $\tau$. b-e, $\mathcal{E}_{\mathrm{NL}}(t, \tau)$ for $\mathcal{E}_{\mathrm{A}}=1.4-5.7 \mathrm{kV} \mathrm{cm}{ }^{-1}$. h-k, $\mathrm{Tw}$-dimensional Fourier transform of $\mathcal{E}_{\mathrm{NL}}(t, \tau)$ from b-e. Labels and circles highlight spectral positions of coherent pump-probe $\left(k_{p 1}, k_{p 2}\right.$, black), four-wave $\left(k_{41}, k_{42}\right.$, red) and six-wave mixing nonlinearities ( $k_{61}$, orange), and arrows visualize wavevectors perpendicular to phase fronts of the respective processes. f,I, $\mathcal{E}_{\mathrm{NL}}(t, \tau)$ and corresponding spectra, respectively, calculated through a microscopic quantum theory for a representative amplitude $\mathcal{E}_{\mathrm{A}}=1.4 \mathrm{kV} \mathrm{cm}{ }^{-1}$. $\mathbf{g}, \mathbf{m}, \mathrm{Same}$ as $\mathbf{f}, \mathbf{I}$ but for a representative amplitude $\mathcal{E}_{\mathrm{A}}=4.3 \mathrm{kV} \mathrm{cm}^{-1}$. Vertical yellow lines in $\mathbf{f}, \mathbf{g}$ indicate the sampling time of $t=3.3 \mathrm{ps}$ for which $\mathcal{E}_{\mathrm{NL}}$ is analysed in Fig. 4 .

interaction. Technically, $a_{\lambda}\left(a_{\lambda}^{\dagger}\right)$ annihilates (creates) an electron in a LL $\lambda$ such that $p_{v}^{\lambda}=\left\langle a_{\lambda}^{\dagger} a_{v}\right\rangle$ identifies both microscopic polarization $(\lambda \neq v)$ and electron distributions $(\lambda=v)$ among different LLs. The terahertz excitation yields an exact quantum dynamics defined by the semiconductor Bloch equations (SBEs; ref. 3)

$$
i \hbar \frac{\partial}{\partial t} p_{v}^{\lambda}=E_{v}^{\lambda} p_{v}^{\lambda}+\sum_{s}\left(\Omega_{s}^{v} p_{s}^{\lambda}-\Omega_{\lambda}^{s} p_{v}^{s}\right)+C_{v}^{\lambda}+D_{v}^{\lambda}
$$

where $E_{v}^{\lambda}$ is the transition energy between two LLs with a dipole $\mathbf{d}_{v}^{\lambda}$, and $\Omega_{v}^{\lambda} \equiv \mathbf{d}_{v}^{\lambda} \cdot \mathcal{E}$ is the Rabi energy of the terahertz field $\mathcal{E}$. The Coulomb interaction renormalizes both $E_{v}^{\lambda}$ and $\Omega_{v}^{\lambda}$, and introduces a contribution $C_{v}^{\lambda}$ whose electron-electron (electron-ion) part contains a repulsive (attractive) Coulomb matrix element $V$ $(W)$ between four LLs (LLs and ions), yielding Coulomb sums of type $\sum_{j, s, u} V_{u, j}^{v, s} p_{u}^{s} p_{j}^{\lambda}$ and $\sum_{j} W_{\lambda}^{j} p_{v}^{j}$. Besides these Hartree-Fock contributions, the SBEs couple to two-particle correlations ${ }^{3,29}$, inducing phonon scattering and excitation-induced dephasing (EID) stemming from Coulomb scattering of polarization with excited electrons $^{3,29,30}$. Longitudinal optical (LO) phonons efficiently dephase polarization involving LLs with energy above $\hbar \omega_{\mathrm{LO}}=36 \mathrm{meV}$, and the EID is described with an excitation-dependent model explained in Supplementary Discussion 10.

The principal quantum number $n$ of a $\operatorname{LL} \lambda=(n, l)$ defines its energy, while an additional quantum number $l$ defines angular momentum and introduces infinite degeneracy among LLs. We use 1,500 LLs in solving equation (1), which is shown to yield a converged macroscopic response (Supplementary Discussion 8). The SBEs then contain $10^{10}$ Coulomb-sum calculations at each time step, rendering simulations extremely demanding. Nevertheless, our computations include even the principal EID effects; at low excitations, all $l$ states are occupied evenly such that the Coulombic inand out-scattering fully compensate each other, yielding vanishing EID. However, strong terahertz excitations generate peaks in the $l$ distribution, unbalancing the Coulomb scattering and yielding detectable EID proportional to the density of excited electrons.

Figure $2 \mathrm{~d}$ compares the transmitted field $\mathcal{E}_{y}$ for $\mathcal{E}_{0}=0.7 \mathrm{kV} \mathrm{cm}^{-1}$ and $5.7 \mathrm{kV} \mathrm{cm}^{-1}$ as obtained from our full theory (solid curves) with the results computed without Coulomb interaction (dashed curves) and a classical calculation with a non-parabolic band and constant $\tau_{c}$ (shaded area). The weak-intensity result $\left(\mathcal{E}_{0}=0.7 \mathrm{kV} \mathrm{cm}^{-1}\right)$ is well reproduced by all theoretical models, verifying the applicability of Kohn's theorem in this case. However, for the stronger field $\mathcal{E}_{0}=5.7 \mathrm{kV} \mathrm{cm}^{-1}$, only the full quantum theory can reproduce the experimental decay of $\mathcal{E}_{y}$ and the redshift of $v_{c}$. As in the experiment, the indicated oscillation minimum of $\mathcal{E}_{y}$ (red vertical line) is delayed by $0.1 \mathrm{ps}$ with respect to the position expected for constant $v_{\mathrm{c}}=1.45 \mathrm{THz}$ (blue vertical line), which manifests a violation of Kohn's theorem. Even though the simplified models neglect Coulomb interaction, they do yield a change of $v_{c}$ implying that the non-parabolic dispersion contributes to the softening of the CR. However, they predict incorrect decay dynamics because the classical computation lacks the phase diffusion among LLs, present in the quantum calculations.

Our full theory also explains the threshold-like onset of dephasing above $\mathcal{E}_{0}=3 \mathrm{kV} \mathrm{cm}^{-1}$ (Fig. 2c, red solid line), attributing this effect to an efficient population transfer. Whereas low terahertz amplitudes $\left(0.7 \mathrm{kV} \mathrm{cm}^{-1}\right)$ excite only a few percent of charge carriers 
into LL $|n=1\rangle$ (Fig. 2e, blue bars), strong pulses drive coherent ladder climbing. At $\mathcal{E}_{0}=4.3 \mathrm{kV} \mathrm{cm}^{-1}$ (violet bars), more than half of the electrons are excited and distributed up to $|n=6\rangle$, where rapid dephasing and relaxation by LO phonon emission sets in. The terahertz-induced population transfer is faster than relaxation such that even LLs above the LO phonon energy are populated. Remarkably, terahertz transients with $\mathcal{E}_{0}=8.7 \mathrm{kV} \mathrm{cm}^{-1}$ (red bars) largely depopulate $|n=0\rangle$ and prepare a compact superposition of eigenstates, peaking at $|n=5\rangle$. This wavepacket rapidly loses its coherence because $25 \%$ of its weight is located above the phonon energy. Thus, the abrupt onset of dephasing above $\mathcal{E}_{0}=3 \mathrm{kV} \mathrm{cm}^{-1}$ arises from the combination of a compact wavepacket distribution and a sharp threshold for phonon scattering. Furthermore, the experiment corroborates that coherent state inversion works even for a massive many-body system and corresponding LLs should be well suited for coherent quantum control.

To test these perspectives systematically, two phase-locked terahertz pulses $\mathrm{A}$ and $\mathrm{B}$, polarized in $x$ - and $y$-directions, respectively, are focused onto the sample for field-resolved twodimensional (2D) terahertz spectroscopy. The transmitted total terahertz field is resolved in amplitude and phase as a function of EOS time, $t$, and the delay between the two incident pulses, $\tau$ (Fig. 3a). Subsequently, the response to individual pulses A and B is subtracted to isolate the nonlinear polarization induced by both pulses $\mathcal{E}_{\mathrm{NL}}(t, \tau)$ (refs 31,32 ), which vanishes for a strictly harmonic CR. In contrast, Fig. 3b-e shows strong nonlinear signals $\mathcal{E}_{\mathrm{NL}}(t, \tau)$ for all peak amplitudes between $\mathcal{E}_{\mathrm{A}}=1.4 \mathrm{kV} \mathrm{cm}^{-1}$ and $5.7 \mathrm{kV} \mathrm{cm}^{-1}$ while $\mathcal{E}_{\mathrm{B}}=90 \mathrm{~V} \mathrm{~cm}^{-1}$ is kept constant. Constant-phase lines of pulse $\mathrm{B}$ appear vertically in the $2 \mathrm{D}$ data maps, whereas the phase fronts of pulse A occur under a $45^{\circ}$ angle (see Supplementary Discussion 3 ).

For $\mathcal{E}_{\mathrm{A}}=1.4 \mathrm{kV} \mathrm{cm}^{-1}$ (Fig. 3b), $\mathcal{E}_{\mathrm{NL}}$ is strongly modulated along $\tau$ with a period $\Delta \tau_{1}=1 / \nu_{\mathrm{c}}=0.69 \mathrm{ps}$, evidencing that the nonlinear interaction is coherently mediated by $v_{\mathrm{c}}$. This feature persists even when the incident pulses do not overlap in time because the coherence is stored in the LL system. For larger $\mathcal{E}_{\mathrm{A}}$ (Fig. 3c-e), an additional modulation with a period of $\Delta \tau_{2}=2 / \nu_{c}=0.34 \mathrm{ps}$, corresponding to twice the cyclotron frequency, emerges and becomes dominant for $\mathcal{E}_{\mathrm{A}}=4.3 \mathrm{kV} \mathrm{cm}^{-1}$. Increasing the field to $\mathcal{E}_{\mathrm{A}}=5.7 \mathrm{kV} \mathrm{cm}^{-1}$ (Fig. 3e) accelerates decoherence, leaving only weak modulations during temporal overlap of both pulses, around $t+\tau=0$. Supplementary Discussion 2 shows similar results for a single-well structure, demonstrating the universality of these nonlinearities.

A 2D Fourier transformation allows us to disentangle different nonlinear optical processes contributing to $\mathcal{E}_{\mathrm{NL}}(t, \tau)$. Figure $3 \mathrm{~h}-\mathrm{k}$ shows the spectral amplitude as a function of the frequencies $v_{t}$ and $v_{\tau}$ associated with the EOS time and the relative delay between the pulses, respectively ${ }^{31,32}$. Several distinct maxima occur at integer multiples of $v_{c}$ : the peak located at $\left(v_{t}, v_{\tau}\right)=\left(v_{c}, 0\right)$ (Fig. 3h, arrow ' $k_{p 1}$ ') represents a pump-probe signal where pulse A (B) acts as a pump (probe) pulse, whereas the maximum at $\left(v_{c},-v_{c}\right)$ (arrow ' $k_{p 2}$ ') is a pump-probe signal for which pulse A and B switch their roles. In addition, strong four-wave mixing (FWM, ' $k_{41}$ ', ' $k_{42}$ ') emerges. As $\mathcal{E}_{\mathrm{A}}$ is increased to $2.9 \mathrm{kV} \mathrm{cm}^{-1}$ (Fig. 3i), the FWM signal at $k_{42}$ (red circle) surpasses the diagonal pump-probe signal $k_{p 2}$ in amplitude. Even six-wave mixing (SWM, ' $k_{61}$ ', orange circle) occurs. For $\mathcal{E}_{\mathrm{A}}=4.3 \mathrm{kV} \mathrm{cm}^{-1}$ (Fig. $3 \mathrm{j}$ ), FWM is the dominant contribution at non-zero $v_{\tau}$ and explains the $\Delta \tau_{2}$-periodic response in the timedomain (Fig. 3d). Finally, the strongly reduced coherence time for $\mathcal{E}_{\mathrm{A}}=5.7 \mathrm{kV} \mathrm{cm}^{-1}$ (Fig. 3k) suppresses wave-mixing processes, and the incoherent pump-probe signal at $k_{p 1}$ dominates.

To identify the origin of these nonlinearities, we apply our many-body theory to the $2 \mathrm{D}$ scenario using the experimental terahertz waveforms. The time-domain data of the full calculation for weak $\left(\mathcal{E}_{\mathrm{A}}=1.4 \mathrm{kV} \mathrm{cm}^{-1}\right.$, Fig. 3f $)$ and strong $\left(\mathcal{E}_{\mathrm{A}}=4.3 \mathrm{kV} \mathrm{cm}^{-1}\right.$, Fig. $3 g$ ) pulses explain our experiment, reproducing the $\Delta \tau_{1}$ - and
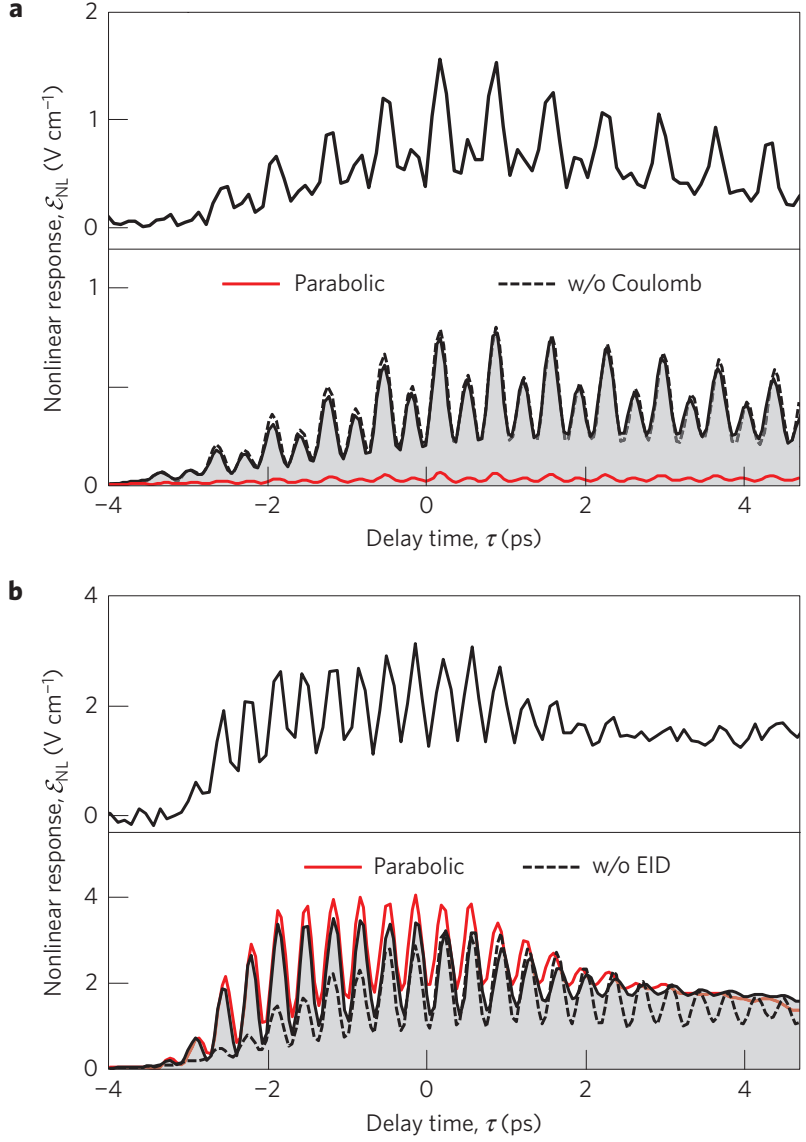

Figure 4 | Time-domain switch-off analysis of nonlinear contributions. a, Nonlinear response $\mathcal{E}_{\mathrm{NL}}(t, \tau)$ for $\mathcal{E}_{\mathrm{A}}=1.4 \mathrm{kV} \mathrm{cm}^{-1}$ at a constant sampling time of $t=3.3$ ps as measured (top panel). Bottom panel: Calculation via a full quantum theory (black curve and shaded area). Dashed curve: calculation excluding Coulomb interaction. Red curve: calculation assuming a parabolic band. $\mathbf{b}, \mathcal{E}_{\mathrm{NL}}(t, \tau)$ for $\mathcal{E}_{\mathrm{A}}=4.3 \mathrm{kV} \mathrm{cm}^{-1}$. Top panel: experiment. Bottom panel: full calculation (black curve and shaded area), calculation assuming a parabolic band (red curve), and calculation neglecting excitation-induced dephasing (dashed curve).

$\Delta \tau_{2}$-periods in $\mathcal{E}_{\mathrm{NL}}(t, \tau)$, as well as their relative weights and decay. Correspondingly, the frequency maps (Fig. 31,m) exhibit multi-wave mixing features in quantitative agreement with the experiment.

The nonlinearities are a consequence of the breakdown of the translational invariance and thus a violation of Kohn's theorem which follows either from the non-parabolic electron dispersion or intrinsic Coulomb-interaction effects. A switch-off analysis allows us to determine the microscopic origin of nonlinearities. Figure 4 shows slices of $\mathcal{E}_{\mathrm{NL}}$ at constant $t=3.3$ ps (see Fig. 3f,g, vertical lines). For $\mathcal{E}_{\mathrm{A}}=1.4 \mathrm{kV} \mathrm{cm}^{-1}$ (Fig. 4a), the $\Delta \tau_{1}$-period dominates the dynamics of $\mathcal{E}_{\mathrm{NL}}(\tau)$. When the non-parabolicity is switched off (red line), $\mathcal{E}_{\mathrm{NL}}$ is strongly suppressed. However, eliminating Coulomb effects (dashed line) yields almost unchanged $\mathcal{E}_{\mathrm{NL}}$, revealing the non-parabolic band structure as the dominant nonlinearity for low fields. For $\mathcal{E}_{\mathrm{A}}=4.3 \mathrm{kV} \mathrm{cm}^{-1}$ (Fig. $4 \mathrm{~b}$ ), the nonlinear response is almost one order of magnitude stronger than for $\mathcal{E}_{\mathrm{A}}=1.4 \mathrm{kV} \mathrm{cm}^{-1}$. Now the parabolic band approximation (red line) produces virtually the same result as the full computation (black line), showing that the Coulomb interaction dominates $\mathcal{E}_{\mathrm{NL}}$ for strong fields. Further insights into the intriguing Coulomb effects are gained by computing $\mathcal{E}_{\mathrm{NL}}(\tau)$ without EID (dashed line, see Supplementary Discussion 10); only the computation with EID explains the experiment, which indicates new possibilities to utilize ultrafast nonlinear switching and decay channels. 
Lifting the protection of Kohn's theorem through nonperturbative excitations of a Landau system opens the door to a rich spectrum of nonlinearities, fine-tuned by the terahertz driving field. High-order nonlinear processes promoted by the intrinsically large dipole moments of the CR hold the prospect for terahertz quantum control, even at low-field amplitudes. Future quantum-logic devices may combine cyclotron transitions with metamaterials to further lower the required field amplitude and the footprint of a single qubit. On-chip electronic terahertz sources may even pave the way towards scalable all-electrical quantum devices. Generally, the principle of accessing internal degrees of freedom in a many-body quantum system through Coulomb correlations suggests that the role of massive many-body interactions, thus far considered detrimental to quantum control, will have to be reassessed.

\section{Methods}

Methods and any associated references are available in the online version of the paper.

\section{Received 1 July 2015; accepted 13 October 2015;} published online 30 November 2015

\section{References}

1. Landau, L. Theory of Fermi-liquids. Sov. Phys. JETP 3, 920-925 (1957).

2. Kohn, W. Cyclotron resonance and de Haas-van Alphen oscillations of an interacting electron gas. Phys. Rev. 123, 1242-1244 (1961).

3. Kira, M. \& Koch, S. W. Semiconductor Quantum Optics (Cambridge Univ. Press, 2011).

4. Huber, R. et al. How many-particle interactions develop after ultrafast excitation of an electron-hole plasma. Nature 414, 286-289 (2001).

5. Chemla, D. S. \& Shah, J. Many-body and correlation effects in semiconductors. Nature 411, 549-557 (2001).

6. Kaindl, R. A., Carnahan, M. A., Haegele, D., Lovenich, R. \& Chemla, D. S. Ultrafast terahertz probes of transient conducting and insulating phases in an electron-hole gas. Nature 423, 734-738 (2003).

7. Gaal, P. et al. Internal motions of a quasiparticle governing its ultrafast nonlinear response. Nature 450, 1210-1213 (2007).

8. Leinß, S. et al. Terahertz coherent control of optically dark paraexcitons in $\mathrm{Cu}_{2}$ O. Phys. Rev. Lett. 101, 246401 (2008).

9. Turner, D. B. \& Nelson, K. A. Coherent measurements of high-order electronic correlations in quantum wells. Nature 466, 1089-1092 (2010).

10. Rice, W. et al. Observation of forbidden exciton transitions mediated by Coulomb interactions in photoexcited semiconductor quantum wells. Phys. Rev. Lett. 110, 137404 (2013).

11. Almand-Hunter, A. et al. Quantum droplets of electrons and holes. Nature 506, 471-475 (2014)

12. Matsunaga, R. et al. Light-induced collective pseudospin precession resonating with Higgs mode in a superconductor. Science 345, 1145-1149 (2014).

13. Noe, G. T. II et al. Giant superfluorescent bursts from a semiconductor magneto-plasma. Nature Phys. 8, 219-224 (2012).

14. Wang, X., Belyanin, A. A., Crooker, S. A., Mittleman, D. M. \& Kono, J. Interference-induced terahertz transparency in a semiconductor magneto-plasma. Nature Phys. 6, 126-130 (2010).

15. Kukushkin, I. V., Smet, J. H., von Klitzing, K. \& Wegscheider, W. Cyclotron resonance of composite fermions. Nature 415, 409-412 (2002).

16. Scalari, G. et al. Ultrastrong coupling of the cyclotron transition of a $2 \mathrm{D}$ electron gas to a THz metamaterial. Science 335, 1323-1326 (2012).

17. Zhang, Q. et al. Superradiant decay of cyclotron resonance of two-dimensional electron gases. Phys. Rev. Lett. 113, 047601 (2014).
18. Arikawa, T. et al. Quantum control of a Landau-quantized two-dimensional electron gas in a GaAs quantum well using coherent terahertz pulses. Phys. Rev. B 84, 241307(R) (2011).

19. Ladd, T. D. et al. Quantum computers. Nature 464, 45-53 (2010).

20. Wieman, C. E., Pritchard, D. E. \& Wineland, D. J. Atom cooling, trapping, and quantum manipulation. Rev. Mod. Phys. 71, S253 (1999).

21. Press, D., Ladd, T. D., Zhang, B. \& Yamamoto, Y. Complete quantum control of a single quantum dot spin using ultrafast optical pulses. Nature 456, 218-221 (2008).

22. Kampfrath, T. et al. Coherent terahertz control of antiferromagnetic spin waves. Nature Photon. 5, 31-34 (2011).

23. Kampfrath, T., Tanaka, K. \& Nelson, K. A. Resonant and nonresonant control over matter and light by intense terahertz transients. Nature Photon. 7, 680-690 (2013).

24. Hu, C. M., Batke, E., Köhler, K. \& Ganser, P. Interaction coupled cyclotron transitions of two-dimensional electron systems in GaAs at high temperatures. Phys. Rev. Lett. 75, 918-921 (1995).

25. Hu, C. M., Batke, E., Köhler, K. \& Ganser, P. Resonant polaron coupling of high index electron Landau levels in GaAs heterostructures. Phys. Rev. Lett. 76, 1904-1907 (1996).

26. Mittendorf, M. et al. Carrier dynamics in Landau-quantized graphene featuring strong Auger scattering. Nature Phys. 11, 75-81 (2015).

27. Al-Jibbouri, H. \& Pelster, A. Breakdown of the Kohn theorem near a Feshbach resonance in a magnetic trap. Phys. Rev. A 88, 033621 (2013).

28. Ghosal, A., Güclü, A. D., Umrigar, C. J., Ullmo, D. \& Baranger, H. U. Correlation-induced inhomogeneity in circular quantum dots. Nature. Phys. 2, 336-340 (2006)

29. Kira, M. \& Koch, S. W. Many-body correlations and excitonic effects in semiconductor spectroscopy. Prog. Quantum Electron. 30, 155-296 (2006).

30. Smith, R. P. et al. Extraction of many-body configurations from nonlinear absorption in semiconductor quantum wells. Phys. Rev. Lett. 104, 247401 (2010)

31. Kuehn, W., Reimann, K., Woerner, M. \& Elsaesser, T. Phase-resolved two-dimensional spectroscopy based on collinear $\mathrm{n}$-wave mixing in the ultrafast time domain. J. Chem. Phys. 130, 164503 (2009).

32. Junginger, F. et al. Nonperturbative interband response of a bulk InSb semiconductor driven off resonantly by terahertz electromagnetic few-cycle pulses. Phys. Rev. Lett. 109, 147403 (2012).

\section{Acknowledgements}

The work in Regensburg was supported by the European Research Council through grant no. 305003 (QUANTUMsubCYCLE) and the Deutsche Forschungsgemeinschaft (LA 3307/1-1, HU 1598/2-1, BO 3140/3-1, and Collaborative Research Center SFB 689). The work at the University of Marburg was supported by the Deutsche Forschungsgemeinschaft through SFB 1083 and grant KI 917/2-2 (M.K.), and the Alexander von Humboldt foundation (J.E.S.).

\section{Author contributions}

T.M., A.B., M.M. and C.L. contributed equally to this work. C.L., T.M., M.K., S.W.K. and R.H. conceived the study. T.M., C.L., A.B., S.B., M.H., T.K., C.S., D.B. and R.H. carried out the experiment and analysed the data. A.B., D.S. and D.B. prepared the sample. M.M., J.E.S., S.W.K. and M.K. developed the quantum-mechanical model and carried out the computations. C.L., T.M., M.M., S.W.K., M.K. and R.H. wrote the manuscript. All authors discussed the results.

\section{Additional information}

Supplementary information is available in the online version of the paper. Reprints and permissions information is available online at www.nature.com/reprints. Correspondence and requests for materials should be addressed to C.L.

\section{Competing financial interests}

The authors declare no competing financial interests. 


\section{Methods}

Experiment. Our sample, grown by molecular beam epitaxy, hosts two 30-nm wide GaAs quantum wells separated by a $10-\mathrm{nm}$ wide $\mathrm{Al}_{0.24} \mathrm{Ga}_{0.76}$ As barrier. In the 2DEGs, a carrier density $\rho_{\mathrm{e}}=1.6 \times 10^{11} \mathrm{~cm}^{-2}$ per $2 \mathrm{DEG}$ is realized by two remote $\delta$-doping layers, symmetrically enclosing the $2 \mathrm{DEG}$ on both sides. $\mathrm{Al}_{0.24} \mathrm{Ga}_{0.76} \mathrm{As}$ spacers of $72 \mathrm{~nm}$ thickness serve as barriers between the 2DEGs and the doping layers (see Supplementary Discussion 2). The resulting high confinement potential for the electrons of $220 \mathrm{meV}$ and the symmetric layout yield two independent electron gases with a high electron mobility of $\mu=4.6 \times 10^{6} \mathrm{~cm}^{2} \mathrm{~V}^{-1} \mathrm{~s}^{-1}$, as verified through van der Pauw measurements.

A Ti:sapphire laser amplifier (centre wavelength: $800 \mathrm{~nm}$, pulse energy: $5.5 \mathrm{~mJ}$, repetition rate: $3 \mathrm{kHz}$, pulse duration: $33 \mathrm{fs}$ ) is used to generate intense few-cycle terahertz fields by tilted-pulse-front optical rectification in a cryogenically cooled $\mathrm{LiNbO}_{3} \mathrm{crystal}^{23}$. In a second optical branch, a small portion of the laser energy drives optical rectification in a $180-\mu \mathrm{m}$-thick (110)-cut GaP crystal. Both terahertz pulses are collinearly focused onto the sample, which is mounted in a magneto-optical cryostat at a constant temperature of $4.3 \mathrm{~K}$. The superconducting magnet provides homogeneous fields which are polarized perpendicularly to the quantum well plane and are tunable between 0 and $5 \mathrm{~T}$. The transmitted terahertz pulses pass a rotatable wire-grid polarizer and are focused onto a 0.5 -mm-thick (110)-cut ZnTe crystal for polarization-resolved electro-optic detection covering the frequency window between 0.1 and $3.0 \mathrm{THz}$. Two mechanical delay stages allow us to independently vary the delay time $\tau$ between the maxima of the two terahertz transients labelled A and B, and the electro-optic sampling time $t$. Differential detection is employed through two mechanical choppers individually modulating the pulses at sub-harmonics of the laser repetition rate, which allows us to clearly isolate feeble terahertz electric fields of less than $0.1 \mathrm{~V} \mathrm{~cm}^{-1}$ from a background of the order of $10 \mathrm{kV} \mathrm{cm}^{-1}$ (see Supplementary Discussion 3 for details).

Theory. We describe Landau electrons in the static magnetic field using the minimal substitution Hamiltonian

$$
\hat{H}=\frac{1}{2 m_{\mathrm{e}}}\left(\widehat{\mathbf{p}}+|e| A_{\text {stat }}(\mathbf{r})\right)^{2}
$$

where $m_{\mathrm{e}}$ is the effective electron mass and $e$ denotes the elementary charge. The vector potential $A_{\text {stat }}$ is employed in the symmetric gauge and contains the magnetic bias perpendicular to the sample surface. The Hamiltonian (2) yields the Landau wavefunctions, cyclotron frequency, and characteristic length scales on which we base our many-body theory. The full many-body Hamiltonian

$$
\hat{H}=\hat{H}_{\mathrm{LL}}+\hat{H}_{\mathrm{ee}}+\hat{H}_{\mathrm{ion}}+\hat{H}_{\mathrm{lm}}
$$

contains the non-interacting contributions $\hat{H}_{\mathrm{LL}}$, into which we incorporate trivial bandstructure effects, and the interaction term $\hat{H}_{\mathrm{lm}}$ describing the light-matter coupling. The complex electron-electron and electron-ion interactions, fully described in Supplementary Discussion 4, are implemented through

$$
\begin{gathered}
\hat{H}_{\mathrm{ee}}=\frac{1}{2} \sum_{\substack{n, l, n^{\prime}, l^{\prime} \\
m, j, m^{\prime}, j^{\prime}}} V_{m^{\prime} j^{\prime}, n^{\prime} l^{\prime}}^{n l, m j} a_{n, l}^{\dagger} a_{m, l}^{\dagger} a_{m^{\prime}, j^{\prime}} a_{n^{\prime}, l^{\prime}} \\
\hat{H}_{\mathrm{ion}}=-\rho_{\mathrm{ion}} \pi r_{\mathrm{c}}^{2} \sum_{n, l, n^{\prime}, l^{\prime}, j} V_{0 j, n^{\prime} l^{\prime} l^{\prime}}^{n l, 0 j} a_{n, l}^{\dagger} a_{n^{\prime}, l^{\prime}}
\end{gathered}
$$

Here, $a_{n, l}^{\dagger}$ and $a_{n, l}$ represent the creation and annihilation, respectively, of Landau electrons with principal quantum number $n$ and angular momentum $l$ in the basis determined through equation (3), $\rho_{\text {ion }}$ is the uniformly distributed positive charge density originating from the dopant ions, and $r_{\mathrm{c}}$ is the radius of the classical Landau orbit. The Coulomb matrix elements $V$ are at the core of the many-body dynamics of the highly excited Landau system and define the numerical challenge, linking electrons in Landau levels through $2.25 \times 10^{6}$ terahertz-induced transition amplitudes. We solve these dynamics via the semiconductor Bloch equations (SBEs; ref. 3) which yield the relevant experimental quantities, such as the polarization and occupation densities, accounting for LO phonon scattering as well as EID, as fully detailed in Supplementary Discussion 10 together with the explicit form of the SBEs. We propagate the related integro-differential equations with a fourth-order Runge-Kutta method, requiring more than $10^{10}$ calculations owing to the Coulomb coupling for each time step, which makes the simulations extremely demanding. Nevertheless, this formidable challenge is executable via an efficient parallel-computing implementation. To study the limitations of Kohn's theorem, we also perform a classical calculation with a non-parabolic energy dispersion and a constant decay, the details of which are given in Supplementary Discussion 4. 